\title{
The US Food and Drug Administration-European Medicines Agency Collaboration in Pharmacovigilance: Common Objectives and Common Challenges
}

\author{
Gerald J. Dal Pan · Peter R. Arlett
}

Published online: 25 December 2014

(C) Springer International Publishing Switzerland (outside the USA) 2014

\begin{abstract}
On 19 February 2014, the US Food and Drug Administration (FDA) and the European Medicines Agency (EMA) announced the formation of a cluster on pharmacovigilance topics. The cluster is designed to complement, and not replace, other international activities in this field. It builds upon years of interactions between the two agencies. The creation of the cluster formalizes this longstanding and productive relationship and facilitates more systematic exchange of information and expertise.
\end{abstract}

\section{Introduction}

On 19 February 2014, the US Food and Drug Administration (FDA) and the European Medicines Agency (EMA) announced the formation of a cluster on pharmacovigilance topics $[1,2]$. The term 'cluster' refers to regular collaborative meetings between the EMA and regulators outside of Europe that focus on specific topic areas that have been identified as requiring an intensified exchange of information and collaboration. The pharmacovigilance cluster builds upon years of interactions between the two agencies. This includes regular teleconferences to discuss post-marketing safety topics of mutual interest and other forms of scientific exchange. The creation of the cluster formalizes this longstanding and productive relationship and facilitates more systematic exchange of information and expertise.

The FDA and the EMA have already set up such clusters to discuss issues related to biosimilars, medicines to treat

\section{G. J. Dal Pan $(\bowtie)$}

US Food and Drug Administration, Silver Spring, MD, USA

e-mail: gerald.dalpan@fda.hhs.gov

P. R. Arlett

European Medicines Agency, London, UK cancer, orphan medicines, medicines for children, and blood-based products, among other topics. Health Canada, the Japanese Pharmaceuticals and Medical Devices Agency, and the Australian Therapeutic Goods Administration are also involved in some of these clusters. The information exchange within the clusters is covered by existing confidentiality arrangements between the two agencies and other participants [3].

\section{Rational for the Cluster}

The pharmacovigilance cluster is designed to complement, and not replace, other international activities in this field. These include activities of the World Health Organization (WHO), the Council of International Organisation of Medical Sciences (CIOMS), the International Conference on Harmonisation of Technical Requirements for Registration of Pharmaceuticals for Human Use (ICH), and the Drug Information Association (DIA). These bodies promote the global importance of post-marketing drug safety and provide a global platform for adverse event data collection (WHO), propose broad frameworks for postapproval safety activities (CIOMS), develop technical specifications for industry submissions to regulatory bodies around the world (ICH), and provide a forum for education and exchange of ideas (DIA). The cluster focuses primarily on product-specific issues, and, to a lesser extent, on practical approaches to monitoring the safety of marketed medicines.

While pharmacovigilance is a global endeavor and each of the two agencies participates in global forums, the rationale for the pharmacovigilance cluster lies in the fact that the two agencies each cover large populations, share a common scientific approach, are often looking at the same 
data, and serve societies with advanced healthcare systems. Because many important and complex product-specific drug safety issues arise virtually simultaneously in Europe and the USA, much of the cluster's work involves timely sharing of information and, more importantly, understanding each other's approach to the specific issue and the basis for each other's decisions and actions. It is important to emphasize that reaching the same conclusion and taking the same action for a particular product-specific drug safety issue is not a goal of the cluster. Many factors, including agency-specific practices and authorities, as well as specifics of different healthcare systems, influence conclusions and decisions.

\section{Frameworks of Post-Approval Drug Safety Monitoring in the USA and Europe}

In the last decade, the importance of post-approval safety monitoring of medicines has been underscored in both the USA and the EU by legislation that has placed specific requirements on both industry and regulators. In September 2007, the US Congress passed, and the President signed, the Food and Drug Administration Amendments Act (FDAAA). Title IX of this act included landmark legislation that granted FDA the authority to require, under certain conditions, post-approval safety labeling changes, safety-related post-approval studies and clinical trials, and risk evaluation and mitigation strategies (REMS) for certain drugs. FDAAA, Title IX also required the FDA to develop a post-market risk identification and analysis system, to screen its adverse event database every 2 weeks and post new safety signals on its website quarterly, and to review post-market safety data systematically for drugs 18 months after approval or after 10,000 patients have taken the drug, whichever is later. The law also required the FDA to establish a consolidated website to provide post-market safety information to patients and providers. The reauthorization of the Prescription Drug User Fee Act, which was also included in FDAAA, provided funding for some of these activities.

In the EU, legislation passed in December 2010 [Directive 2010/84/EU and Regulation (EU) No. 1235/2010], accompanied by an implementing regulation in June 2012 [Commission Implementing Regulation (EU) No. 520/2012 of 19 June 2012] and further amended in 2012 [Directive 2012/26/EU and Regulation (EU) No. 1027/2012] strengthened the pharmacovigilance system [4]. This was achieved by providing a clear legal basis for different roles and responsibilities of the principal actors within the EU (notably the pharmaceutical industry and regulators), directing the EMA to have robust and rapid decision making, increasing engagement of patients and practitioners, requiring science-based integration of benefit and risk, ensuring that requirements are proportionate to risk, focusing on more proactivity and planning, reducing duplication within the system and increasing efficiency, and increasing transparency. Specific changes included strengthened coordination at the EMA, strengthened authorization requirements with increased focus on pharmacovigilance, a requirement for risk management plans for all new medicines, stronger requirements for studies in the post-authorization phase of safety and efficacy, measurement of the effectiveness of risk minimization, new requirements for each member state to introduce patient reporting of adverse events, a requirement for companies to submit post-market benefit-risk reports, the creation of the Pharmacovigilance and Risk Assessment Committee, coordination of pharmacovigilance inspections and audits across the EU, and better funding via fees [5].

Although there are many differences in the details of the recently passed laws between the USA and the EU, there are certain underlying common themes: an increased emphasis on transparency, a higher level of accountability, a rigorous scientific basis for post-marketing decision making, improved processes, and better funding for these activities. These shared values provide an obvious starting point for close collaboration.

The legislation in each jurisdiction that strengthened post-marketing drug safety did so in a way that went beyond the harmonized ICH agreements of the late 1990s and early 2000s. For example, a risk management plan in the EU and risk evaluation and mitigation strategy in the USA are two quite different approaches to managing the risk of a medicine. Pharmacovigilance planning is emphasized in the EU system, while a similar approach has not been formally adopted in the USA. The detailed framework of post-approval safety studies and postapproval efficacy studies is different from that of postmarketing requirements for studies or clinical trials of a safety issue in the USA. There are also fundamental operational differences between the two agencies. In the EU system, scientific review is performed by experts within a decentralized network of national agencies, whose work is referred to a centralized committee, the Pharmacovigilance and Risk Assessment Committee. In the USA, scientific review work is conducted within a single centralized agency, though there is no single body within the FDA that reviews each and every recommendation.

\section{Conclusion}

Through careful bilateral planning, the cluster is able to promote information sharing and rigorous scientific exchange without pressure to arrive at an identical decision 
about any particular issue. Indeed, the differences in the regulatory frameworks of the two systems may lead to one decision in the EU and another in the USA, even when the scientific conclusions are similar.

Disclaimer The views expressed in this article are the personal views of the authors and may not be understood or quoted as being made on behalf of or reflecting the position of the US FDA or the EMA or one of its committees or working parties.

Funding and conflict of interest No sources of funding were used to assist in the preparation of this manuscript. Gerald J. Dal Pan and Peter R. Arlett have no conflicts of interest that are directly relevant to the content of this manuscript.

\section{References}

1. US Food and Drug Administration. FDA and European Medicines Agency strengthen collaboration in pharmacovigilance area. [Press release; 19 Feb 2014]. Available at: http://www.fda.gov/news events/newsroom/pressannouncements/ucm386372.htm. Accessed 20 Dec 2014

2. European Medicines Agency. European Medicines Agency and US Food and Drug Administration strengthen collaboration in pharmacovigilance area. [Press release; 19 Feb 2014]. Available at: http://www.ema.europa.eu/ema/index.jsp?curl=pages/news_and_ events/news/2014/02/news_detail_002028.jsp\&mid=WC0b01ac0 58004d5c. Accessed 20 Dec 2014

3. http://www.ema.europa.eu/ema/index.jsp?curl=pages/partners_and_ networks/document_listing/document_listing_000228.jsp\&mid=WC0 b01ac058003176e\#section1. Accessed 20 Dec 2014

4. European Medicines Agency. Pharmacovigilance legislation. Available at: http://www.ema.europa.eu/ema/index.jsp?curl= pages/special_topics/general/general_content_000491.jsp\&mid= WC0b01ac058058f32d. Accessed 20 Dec 2014

5. Arlett P, Portier G, de Lisa R, Blake K, Wathion N, Dogne JM, Spooner A, Raine J, Rasi G. Proactively managing the risk of marketed drugs: experience with the EMA Pharmacovigilance Risk Assessment Committee. Nat Rev Drug Discov. 2014;13(5):395-7. 